\title{
HOW PEOPLE EVALUATE A PRODUCT IN AN ONLINE ENVIRONMENT: THE ROLE OF UNCERTAINTY AND LIKING FEELING
}

\author{
Francesca Checchinato, Department of Management - Ca' Foscari University of Venice, Italy \\ f.checchinato@unive.it
}
Isabella Procidano, Department of Management - Ca' Foscari University of Venice, Italy
Isabella.procidano@unive.it

Marta Pisani, Department of Management - Ca' Foscari University of Venice, Italy

marta.pisani89@gmail.com

\section{Introduction}

Although it is well known that travellers look at other travellers' opinions and recommendations to plan their trips, and the number of people reviewing touristic attractions is increasing, little is known about how people judge them. Numerous studies have examined user behaviour in creating contents and participating in the on-line community using various methods, but research that investigates how consumers express opinions about hotel attributes is still lacking. In particular, since expressed preferences as well as ratings, could be the result of both the uncertainty of consumers in evaluating an item and the liking feeling about the item (D'Elia \& Piccolo, 2005), the aim of this paper is to understand the role of these components in the evaluating process, in order to segment the market and to define different users' profile.

\section{Background}

Previous research about user generated contents (hereafter, UGC) and the rating sites can be spitted into two main topics: 1) research related to the use of UGC as a source of information for other consumers and 2) research aimed at understanding contributors, in order to explain their behaviour and characteristics. In this research we focus on the latter point.

In order to understand reviewers' behaviour, it is important to analyse "why", "how" and "what" users evaluate in travel websites such as TripAdvisor. The three questions could be related to each other, since the reasons why users evaluate an object could affect the way a review is written.

Most of the previous studies attempt to understand why people are willing to share their information and contribute their knowledge for free, as it happens in the rating sites such as TripAdvisor, Expedia, etc.. McLure, Wasko and Faraj (2000) analysed why people provide knowledge in communities of practice. Some results might be helpful for rating sites as they state that people believe that sharing information is "the right thing to do". Yang and Lai (2011) showed that internal self-concept-based motivation significantly influences the knowledge-sharing intention of Wikipedia contributors. Park, Jung and Lee (2011) demonstrated that ego-involvement is highly associated with both the attitude toward uploading video content online and the intention to upload. So motivations are related both to social and personal reasons. Other scholars analyse barriers faced in the online content creation (so, the "why not"), finding that some users prefer to participate in communities asking questions rather than adding contents because they need to know more before creating their own contents (Preece, Nonnecke, \& Andrews, 2004). One of the main reasons not to create contents is related to the fact that users are not sure if their knowledge is important, accurate or relevant to specific topics, so their feelings of incompetency become a barrier to share opinions. In the travel sector time constrictions seem to be the main reason for not contributing, but also a lack of experience has been mentioned (Gretzel, Yoo, \& Purifoy, 2007).

An important factor that affects evaluation process is the consumer uncertainty: usually analysed in the buying process, it is related to the difficulty of comparing options and choosing the right 
product (Broniarczyk \& Griffin, 2014). Recently, consumer uncertainty has been analysed in the evaluation and expression of preferences through ratings context; some research (D'Elia \& Piccolo, 2005; Piccolo \& D'Elia, 2008) shows that consumer uncertainty adds up to the basic feeling about an item. It was also found that splitting the consumer evaluation into two components - uncertainty and liking feeling - can be helpful in explaining how users evaluate an object, such as a hotel. This model was adopted to explain users' responses during survey, but since users express (ordinal) preferences also volountarely, providing reviews and rates in travel website such as TripAdvisor, we test the model in this new contex in order to verify the theoretical assumptions.

In the rating sites, consumers share their opinions in the form of 1) qualitative descriptions of experiences and 2) ratings. The latter one could be considered the numerical expression of customer satisfaction, a post-purchase evaluative judgment. Therefore considering how the evaluation process might affect the ratings, we test if in voluntary contexts - where consumers decide to judge an item and at the same time he/she can read how previous consumers have judged it - the incertainty components still exist. In particular we assume:

Hypothesis 1: in UGC context, in addition to the liking feeling, the evaluations expressed

by users also depend on consumer uncertainty.

Moreover, from the previously mentioned research about motivations and contributors' characteristics in the UGC, we posit that the level of user experience could affect the evaluation process, since high levels of experience reduce barriers to adding reviews and, at the same time, they improve user competences in judging items. The same assumptions are drawn from the classical theory of consumer behaviour that points out that customer satisfaction is influenced by the customers' background (Broniarczyk \& Griffin, 2014), among the others. So we test:

Hypotesis 2: the more is the experience in evaluating and rating touristic attractions, the more the liking feeling influences the final review, since the uncertainty component becomes lower.

We also would verify whether Tripadvisors contributors can be clustered in distinct profiles based on the uncertainty and liking feeling components and on their main travel purpose: leisure or business. The latter has been prove to be a factor that affect consumer expectations and so the satisfaction. As suggested by Yavas and Babakus (2005) hoteliers should conduct guests satisfaction for the two clusters.

\section{Research method}

The more useful statistical tool for analysing preference/evaluation is the mixture model proposed by D'Elia and Piccolo (2008), known as CUB, i.e. Covariate Uniform Binomial, a generalization of MUB, Mixture Uniform Binomial (D'Elia \& Piccolo 2005). This model is characterized by a significant flexibility because the covariates of raters are used in order to define and estimate heterogeneity in the evaluation of the perceived quality due to the same covariates. Regarding CUB $(0,0)$ (the MUB model) liking/feeling and uncertainty are represented by a linear combination, Y, of two different random variables: a shifted Binomial and a Uniform; so, Y describes the process that leads a respondent to express the final rating:

$$
P(Y=y)=\pi\left[\left(\begin{array}{c}
m-1 \\
y-1
\end{array}\right)(1-\xi)^{p-1 \xi \xi i-y}\right]+(1-\pi) \frac{1}{m}
$$

where $y=1,2, \ldots, m, m>3$ is a discrete set of values of an hedonic scale; $\xi_{c}[0,1]$ and $\pi c[0,1]$ are the parameters characterizing the shape of the probability distribution of the mixture model. The model postulates the existence of two subgroups of raters, whose evaluation is characterized by a different weight assigned respectively to the component of liking/feeling and uncertainty: the informed/thoughtful subgroup and the uninformed/instinctive one. In such a way, the coefficients $\pi$ and $(1-\pi)$ can be interpreted, respectively, as a measure of their proportion in the population. In the 
evaluation process $(1-\pi)$ represents a measure of uncertainty ( 1 maximum uncertainty, 0 no uncertainty), while (1- $\xi$ ) measures the liking/feeling ( 0 disliking, 1 maximum rating).

To test our hypotheses, we focused our research on hotel reviews on TripAdvisor, a leader website in the travel industry. Since hotel ratings also depend on the destination (Bulchand-Gidumal, Melián-González, \& González Lopez-Valcarcel, 2013), we analyse hotel reviews in a single destination, Venice, that is an important touristic destination of the world. Moreover, we restricted our analysis to four-star hotels in order to have a (presumably) standard quality about the rated items. For each hotel, the first ten reviews (the first page of the hotel page) were considered. For each review, we collected the rating (from 1 to 5) for the considered hotel, the trip-purpose (family, couple, friends, business or solo) and the user profile descriptors - number of reviews, number of hotel reviews, number of cities for which reviews are made, and number of helpful votes provided by other TripAdvisor members for the written reviews.

\section{Results and discussion}

A total of 731 reviews were collected and used for our analysis. The average rate is high (4.273) as expected from four-star hotels. In Table 1 the estimated parameters for a CUB $(0,0)$ are reported and the values of AIC and ICON (Information Content) are showed in order to evaluate the goodness of fit. Moreover the normalized dissimilarity index (Diss) is reported: only $2 \%$ of units should be moved among the categories in order to get a perfect fit.

Table 1 - CUB $(0,0)$ model summary

\begin{tabular}{cccccc}
\hline $\mathbf{1 - \pi}$ & $\mathbf{1 -} \boldsymbol{\xi}$ & $\mathbf{( 1 - \boldsymbol { \pi } ) / \mathbf { m }}$ & AIC & ICON & DISS \\
\hline $\mathbf{0 . 1 3 3}$ & 0.867 & 0.027 & 1316.041 & 0.28 & 0.02 \\
$\mathbf{( 0 . 0 2 6 )}$ & $(0.009)$ & $(0.005)$ & & & \\
\hline \multicolumn{5}{c}{ Standard error is reported within brackets . }
\end{tabular}

Then a CUB $(1,1)$ model with covariate (reviews' number for each reviewer) was estimated in order to investigate if this variable, as a proxy of users' experience, impacts on the respondents' ratings. On the basis of the results we can argue that CUB $(1,1)$ performs better than the model without covariates (lower AIC (1308.07) and higher ICON (0.29)). The estimated parameters from CUB $(1,1)$ are statistically significant and are used in order to calculate the uncertainty and the liking/feeling for each respondent. So we can state that the uncertainty plays a role in consumers' evaluation process and can be adds up to the basic liking feeling. Therefore hypothesis 1 is supported.

The Figure 1 represents respectively uncertainty and liking/feeling versus the number of reviews carried out by raters. The higher is the number of reviews, the lower is the level of uncertainty in the evaluation. At the same time, the influence of the liking/disliking feeling becomes higher since the ranking gets lower, it means that after evaluating and "consuming" a high number of touristic attractions, consumers become more critical. Therefore, also hypothesis 2 is supported postulating a relevant role played by experience in customer evaluations.

Figure 1 Uncertainty and liking/feelings vs Number of reviews
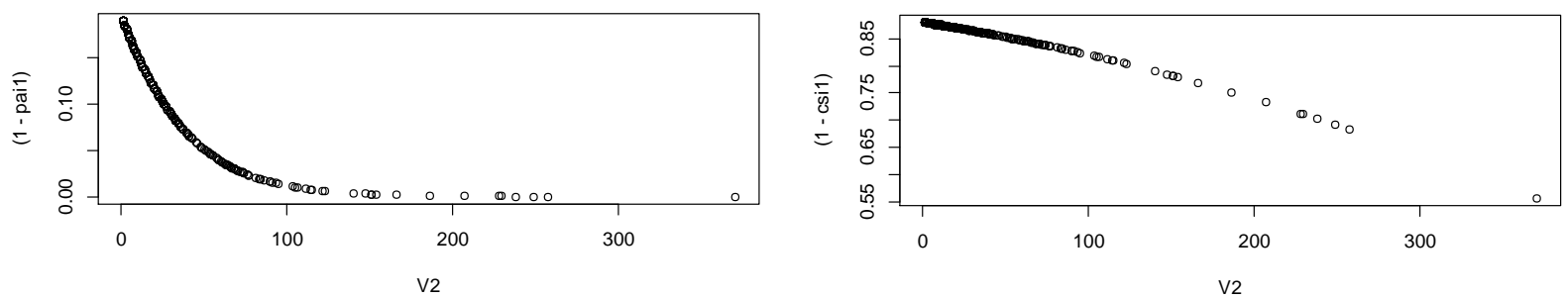
Morevoer, in order to identify users with similar expectations (related to the purpose trip) we performed a cluster analysis using uncertainty and liking/feeling estimated by the $\mathrm{CUB}(1,1)$ as clustering variables.

The Euclidean distance and K-means method were used. Four groups are obtained on the basis of higher silhouette index (equal to 0.5). In the Table 2 the average of all variables for each group $(\mathrm{C} 1, \mathrm{C} 2, \mathrm{C} 3, \mathrm{C} 4)$ and for the whole sample are reported. The variable for profiling the clusters regarding the trip-purpose declared by reviewer. In TripAdvisor, reviewers get different types of star badges based on the number of reviews they have posted, our cluster analysis supports this division, adding components related how reviewers evaluate a touristic attraction. Users with low experience ( $\mathrm{C} 1$ and $\mathrm{C} 4$ in our anlaysis, from white to green star badges in TripAdvisor clusters) have the higher level of uncertainty and liking/feeling. It is interesting to point out that in $\mathrm{C} 1$ cluster $72.3 \%$ of the reviews are about couple travel. Cluster C2 might be associated to TripAdvisor super contributors (gold star badge): they have the lower level of uncertantly and liking/feeling, in this group we can find the higher percentage of reviews of people who travel for business and with friends. Cluster C3 has the higher percentage of rates about travel with family experiences.

Table 2 - Clustering and profiling variables: average and relative frequency

\begin{tabular}{lccccc}
\hline & $\mathrm{C} 1$ & $\mathrm{C} 2$ & $\mathrm{C} 3$ & $\mathrm{C} 4$ & Whole sample \\
\hline Rate & 4.234 & 4.178 & 4.298 & 4.320 & 4.273 \\
Number of reviews & 13.010 & 100.397 & 30.742 & 3.214 & 24.345 \\
Business & 0.088 & 0.164 & 0.097 & 0.060 & 0.089 \\
Friends & 0.075 & 0.123 & 0.056 & 0.102 & 0.087 \\
Couple & 0.723 & 0.548 & 0.621 & 0.651 & 0.651 \\
Family & 0.100 & 0.137 & 0.202 & 0.163 & 0.150 \\
Solo & 0.012 & 0.027 & 0.024 & 0.023 & 0.021 \\
1-pai1 & 0.141 & 0.022 & 0.089 & 0.18 & 0.129 \\
1-csi1 & 0.874 & 0.817 & 0.865 & 0.879 & 0.867 \\
Size (number of statistical units) & 159 & 73 & 124 & 215 & 571 \\
\hline
\end{tabular}

Even if the trip-purpose does not explain the uncertainty and liking feeling, it helps to understand the clusters and which hotels' travellers are more used to write a review.

In this research we demonstrate that also in a voluntary context, rates depend also by uncertainty and the experience plays a role in reducing it. Segmentation helps hoteliers to define different strategy to cope with the different guests profile. Our findings suggest that affecting experienced customers' opinions is more difficult for hotel managers because they are not uncertain. Experienced evaluators tend to be more critical about the hotel service, maybe because they better know what they want, so hoteliers should find the way to know their opinion during their stay in order to drive the right expectations and to find solutions for unsatisfactory situations. Moreover our findings show that "how" reviewers evaluate touristic attractions might be helpful in order to define clusters, contributing to enhance our knowledge about Internet users' behavior and patterns. Understanding the level of liking/feeling and uncertainty of customers - and not only which attributes they value - can help hotel managers to better know their customers and adopt incentives to stimulate reviews when they face travellers that stay in their hotel in couple and not for business reasons. By understanding the users' concerns and the related level of uncertainty, managers can ultimately define effective strategies and enhance their impact. Online and offline promotions might be developed in order to reach the different groups.

This is the first step of a wide research that aim at analysing how people create online content. Adding more destinations, hotels and turistic attractions' categories, and rating websites it will be possible to enhance our comprehension of how users evaluate items. Moreover adding information about what reviewers evaluate might contribute to the understanding of their behaviour.

References: available upon request 